\title{
Ectopic expression of KCNE3 accelerates cardiac repolarization and abbreviates the $Q T$ interval
}

\author{
Reza Mazhari, ${ }^{1,2}$ H. Bradley Nuss, ${ }^{1,2}$ Antonis A. Armoundas, ${ }^{1,2,3}$ Raimond L. Winslow, ${ }^{2,3}$ \\ and Eduardo Marbán 1,2,3 \\ ${ }^{1}$ Department of Medicine, \\ ${ }^{2}$ Institute of Molecular Cardiobiology, and \\ ${ }^{3}$ Whitaker Biomedical Engineering Institute and Center for Computational Medicine and Biology, \\ Johns Hopkins University, Baltimore, Maryland, USA \\ Address correspondence to: Eduardo Marbán, Institute of Molecular Cardiobiology, Johns Hopkins School of Medicine, \\ 720 Rutland Avenue, Ross Building 844, Baltimore, Maryland 21205, USA. \\ Phone: (410) 955-2776; Fax: (410) 955-7953; E-mail: marban@jhmi.edu.
}

H. Bradley Nuss's present address is: Cardiology Research Group, University of Maryland School of Medicine, Baltimore, Maryland, USA.

Received for publication January 16, 2002, and accepted in revised form March 11, 2002.

\begin{abstract}
Regulatory subunit KCNE3 (E3) interacts with KCNQ1 (Q1) in epithelia, regulating its activation kinetics and augmenting current density. Since E3 is expressed weakly in the heart, we hypothesized that ectopic expression of E3 in cardiac myocytes might abbreviate action potential duration (APD) by interacting with $\mathrm{Q} 1$ and augmenting the delayed rectifier current $\left(\mathrm{I}_{\mathrm{K}}\right)$. Thus, we transiently coexpressed E3 with Q1 and KCNE1 (E1) in Chinese hamster ovary cells and found that E3 coexpression increased outward current at potentials by $\geq-80 \mathrm{mV}$ and accelerated activation. We then examined the changes in cardiac electrophysiology following injection of adenovirus-expressed E3 into the left ventricular cavity of guinea pigs. After 72 hours, the corrected QT interval of the electrocardiogram was reduced by $\sim 10 \%$. APD was reduced by $>3$-fold in E3transduced cells relative to controls, while E-4031-insensitive $I_{K}$ and activation kinetics were significantly augmented. Based on quantitative modeling of a transmural cardiac segment, we demonstrate that the degree of QT interval abbreviation observed results from electrotonic interactions in the face of limited transduction efficiency and that heterogeneous transduction of E3 may actually potentiate arrhythmias. Provided that fairly homogeneous ectopic ventricular expression of regulatory subunits can be achieved, this approach may be useful in enhancing repolarization and in treating long QT syndrome.
\end{abstract}

J. Clin. Invest. 109:1083-1090 (2002). DOI:10.1172/JCI200215062.

\section{Introduction}

Long QT (LQT) syndrome is associated with cardiac arrhythmias and sudden cardiac death (1). LQT syndrome is characterized by QT interval prolongation on the electrocardiogram (ECG). This syndrome can be familial, as either an autosomal recessive or a dominant trait, or acquired (1). While myocardial ischemia and cardiomyopathy are among the heart diseases that cause LQT syndrome, use of medications such as antibiotics can also lead to it (2). Mutations in genes encoding ion channels, leading to abnormal channel function, are the main cause of the heritable form of this disorder (3). Among these genes are pore-forming potassium channel $\alpha$ subunits KCNH2 (encoding HERG) and KCNQ1 (encoding KvLQT1) (3). KCNH2 and KCNQ1 (Q1) are responsible for rapidly activating delayed rectifier potassium currents $\left(\mathrm{I}_{\mathrm{Kr}}\right)$ and slowly activating delayed rectifier potassium currents $\left(\mathrm{I}_{\mathrm{Ks}}\right)$, respectively. While the majority of mutations linked to LQT syndrome are associated with $\alpha$ subunits, genetic lesions in accessory subunits, such as the KCNE family of peptides, also contribute to LQT syndrome. For example, mutations in both KCNE1 (4) (encoding MinK) and KCNE2 (5) (encoding MinK-related peptide 1 [MiRP1]), which regulate $\mathrm{I}_{\mathrm{Ks}}$ and $\mathrm{I}_{\mathrm{Kr}}$ in the heart, respectively, have been linked to LQT syndrome in patients. Unfortunately, therapeutic approaches for correction of electrical abnormalities associated with LQT syndrome are limited and not very effective.

Recently, Schroeder et al. (6) reported that KCNE3 (encoding MiRP2) could coassemble with Q1 in cultured cells and result in a rapidly activating, constitutively open channel. KCNE3 (E3) is abundantly expressed in skeletal muscle (7), kidney, and intestine (8), while it is weakly expressed (if at all) in the heart $(6,9)$. It has been postulated that $\mathrm{E} 3$ has a role in regulating chloride secretion in epithelia through cystic fibrosis transmembrane conductance regulators (10). Abbott et al. showed that E3 plays a major role in regulating skeletal muscle function, and mutations in E3 are associated with periodic paralyses (7). Since there 
is a low abundance of E3 in the cardiac tissue, we hypothesized that ectopic expression of $\mathrm{E} 3$ in cardiac myocytes might abbreviate action potential duration (APD) by interacting with Q1 and augmenting the delayed rectifier current. Our results show that adenoviral gene delivery of $\mathrm{E} 3$ results in an increase in outward current, abbreviates APD in cardiac myocytes, and shortens the QT interval of the ECG in transduced animals. However, quantitative modeling points out potential pitfalls of such an approach if transduction is inhomogeneous.

\section{Methods}

The work presented was performed in accordance with $\mathrm{NIH}$ guidelines for the care and use of laboratory animals and the guidelines of the Animal Care and Use Committee of the Johns Hopkins University.

Plasmid and adenovirus construction. Human KCNE3 cDNA (GenBank accession no. AF302494) was obtained by RT-PCR (GIBCO BRL; Invitrogen, Carlsbad, California, USA) from human RNA and cloned into the multiple cloning site of the expression vector pCGI (11) (pCGI-KCNE3) and adenovirus shuttle vector AdCGI (AdCGI-KCNE3). Both of these vectors are bicistronic constructs (through an internal ribosome entry site) driven by a cytomegalovirus promoter and carrying green fluorescent protein (GFP) as a reporter. Detailed methods of adenovirus vector construction have been described previously (12-14). Briefly, adenovirus vectors were generated using Crelox recombination of purified $\Psi 5$ viral DNA and shuttle vector AdCGI-KCNE3 (13). The recombinant vectors were plaque-purified, expanded, and purified using cesium chloride gradients, yielding concentrations of about $8 \times 10^{10}$ plaque-forming units (PFUs) per milliliter.

Transient transfections. Chinese hamster ovary $(\mathrm{CHO})$ $\mathrm{K} 1$ cells (American Type Culture Collection, Manassas, Virginia, USA) were transiently transfected with KCNQ1 (kindly provided by M. Sanguinetti, University of Utah, Salt Lake City, Utah, USA) or KCNQ1KCNE1 (kindly provided by G.F. Tomaselli, Johns Hopkins University) and PCGI-KCNE3 (when indicated), with $1 \mu \mathrm{g}$ total cDNA using Lipofectamine-Plus (GIBCO BRL; Invitrogen), following the manufacturer's instructions. GFP-positive cells were studied within 24-36 hours of transfection.

In vivo and ex vivo transductions. Adenovirus (AdCGIKCNE3; 90-100 $\mu$ l, equivalent to $\sim 10^{9}$ PFUs) was injected, at a rate of about $50-80 \mu \mathrm{l} / \mathrm{s}$ using a 30 -gauge needle, in the left ventricular cavity of isoflurane-anesthetized female guinea pigs (280-340 g), while the aorta and pulmonary artery were clamped for 50-60 seconds (15). This resulted in 10-20\% cardiomyocyte transduction efficiency in the left ventricular wall as determined by fraction of green cells after enzymatic cell isolation. In some experiments, as indicated, freshly isolated rabbit left ventricular myocytes were transduced in vitro using the same virus, while cultured in
M199 media with 2\% FBS, 1\% streptomycin/penicillin (all GIBCO BRL; Invitrogen). The myocytes were washed with virus-free medium after 1 hour and incubated at $37^{\circ} \mathrm{C}$ and $5 \% \mathrm{CO}_{2}(16)$.

Current and action potential recordings. Sixty-five to seventy-two hours after in vivo injection, myocytes from the left ventricles of guinea pigs were isolated using enzymatic digestions as described previously (12). Action potentials were recorded from the ex vivo transduced rabbit cells within 24-36 hours after transduction. Membrane currents and action potentials were recorded using whole-cell patch clamp, in GFP-positive myocytes. Cells were bathed in solution containing (in mmol/l): $140 \mathrm{NaCl}, 5.4 \mathrm{KCl}, 1 \mathrm{MgCl}_{2}, 10$ HEPES, 10 glucose, pH 7.4 (adjusted with $\mathrm{NaOH}$ ). For current or action potential recordings, 0.1 or $2 \mathrm{mmol} / \mathrm{l}$ $\mathrm{CaCl}_{2}$, respectively, was added to the bath solution. For delayed rectifier potassium current $\left(\mathrm{I}_{\mathrm{K}}\right)$ recordings, 0.2 $\mathrm{mmol} / 1 \mathrm{CdCl}_{2}$ and $0.5 \mathrm{mmol} / 1 \mathrm{BaCl}_{2}$ were added to the bath solution; $\mathrm{I}_{\mathrm{Kr}}$ was blocked, as indicated, with 5 $\mu \mathrm{mol} / \mathrm{l}$ E-4031. Sodium currents were inactivated in the native cells by using holding potentials of $-40 \mathrm{mV}$. Borosilicate glass pipettes were pulled and fire-polished to final tip resistances of 4-5 $\mathrm{M} \Omega$ when filled with pipette solution containing (in $\mathrm{mmol} / \mathrm{l}$ ): 140 K-glutamate, $1 \mathrm{MgCl}_{2}$, 10 HEPES, 5 EGTA, 4 MgATP, $\mathrm{pH} 7.3$ (adjusted with $\mathrm{KOH}$ ). This resulted in a liquid junction potential of $-18 \mathrm{mV}$; here, results are not corrected for this value, unless stated otherwise. Uncompensated capacitance currents in response to small hyperpolarizing voltage steps were recorded for offline integration as a means of measuring cell capacitance. All CHO-cell recordings were obtained at room temperature, while experiments on cardiomyocytes were performed at $37^{\circ} \mathrm{C}$.

Voltage protocols used in this study are described in the text where necessary; action potentials were initiated by a short depolarizing current pulse ( $2 \mathrm{~ms}, 100-300$ $\mathrm{pA}, 10-15 \%$ over the threshold) using the voltage-follower mode while sampling at $2 \mathrm{kHz}$.

ECGs. Body surface ECGs were recorded within 2 hours after operation (baseline) and 65-72 hours (day 3) after adenovirus injection, under similar conditions, as previously described (12). Measured QT intervals were corrected (QTc) for heart rate using the square-root method as described previously (17).

Mathematical modeling. A detailed description of the model of electrical propagation in ventricular tissue has been published previously (18). The geometry of the integrative model used here was assumed, for simplification, to be a three-dimensional fiber $(3 \times 70 \times 3$ nodes, with $0.2 \mathrm{~mm}$ resolution), representing a transmural segment of the left ventricular wall (see Figure 6a). At each node, an experimentally derived detailed biophysical model of the cardiac action potential was used to represent each myocyte (19). $\mathrm{I}_{\mathrm{Ks}}(20)$, sodiumcalcium exchanger current (21), transient outward currents (20), and inward rectifier currents (20) were altered, based on previously published experimental 


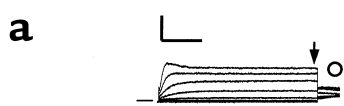

Q1

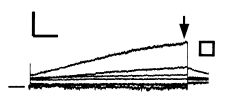

Q1/E1

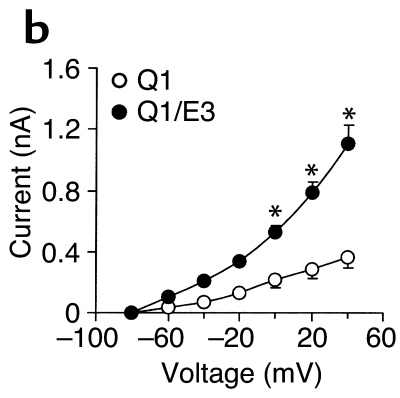

Figure 1

KCNQ1-KCNE3 coexpression in $\mathrm{CHO}$ cells. (a) Original current traces of KCNQ1 alone (Q1), KCNQ1-KCNE3 (Q1/E3), KCNQ1-KCNE1 complex (Q1/E1), and KCNQ1/KCNE1/KCNE3 (Q1/E1/E3) recorded with 2-second (upper panels) or 4-second (lower panels) voltage steps from -80 to $40 \mathrm{mV}$, followed by a repolarization to $-50 \mathrm{mV}$ for 0.3 seconds. Vertical scale bars $=0.2 \mathrm{nA}$; horizontal scale bars $=0.5$ seconds; dash marks designate zero current. (b and c) Currents at the end of depolarizing pulses (arrows in left panels of a) for Q1 alone (open circles, $n=4$ ), Q1/E3 (filled circles, $n=4$ ), Q1/E1 (open squares, $n=3$ ), and Q1/E1/E3 (filled squares, $n=4$ ).

data, with a smooth transition between the layers, to simulate in vivo action potential morphology and duration accurately (see Figure 6, a and b). This model resulted in transmural repolarization time (activation time + time to $90 \%$ repolarization) and APD patterns that match in vivo findings accurately (see Figure 6b). Ionic current associated with Q1-E3 coexpression, based on experimental observations, was added as a new current in a certain percentage of the nodes within the geometry, to simulate the approximate observed transduction efficiency (see Figure 6a). For solutions as a function of time, the associated differential equations were solved using parallel computational methods, with three $375-\mathrm{MHz}$ processors for 400 milliseconds simulation time to capture activation and repolarization (18).

Statistical analysis. All the data shown are mean \pm SEM. ANOVA was used for statistical analysis along with post-hoc analyses taking into consideration repeated measurements, and $P<0.05$ was considered to indicate statistical significance.

\section{Results}

KCNE3-KCNQ1 coassembly. To determine the effects of $\mathrm{E} 3$ on $\mathrm{Q} 1$ functional properties, we transiently transfected CHO cells with Q1 alone, Q1 and E3, or Q1, E3, and KCNE1 (E1). Compared with Q1 alone, addi- tion of E3 increased the rate of activation; outward currents were augmented by an average of 4.8 -fold at all voltages higher than $-60 \mathrm{mV}(P<0.05)$ (Figure 1, $a$ and $b$ ). In native cells, it is believed that the Q1-E1 complex forms $\mathrm{I}_{\mathrm{Ks}}(22)$; thus we tested the effects of $\mathrm{E} 3$ on Q1, coexpressed with E1. As in the absence of $\mathrm{E} 1$, addition of $\mathrm{E} 3$ resulted in currents that activated quickly and remained open throughout the pulse (Figure 1a). In addition, E3 coexpression resulted, on average, in a 2.8 -fold higher current measured at the end of the activation pulse at voltages higher than $80 \mathrm{mV}(P<0.05)$ (Figure 1c). Addition of E3 also resulted in an acceleration in deactivation compared with Q1, with or without E1 present (Figure 1a). Moreover, there was no difference in currents associated with Q1-E3 coassembly, with or without E1 present. For example, potassium current at the end of the test pulse to $40 \mathrm{mV}$ was $1.1 \pm 0.1 \mathrm{nA}$ for Q1-E3, compared with $1.1 \pm 0.3 \mathrm{nA}$ for $\mathrm{Q} 1-\mathrm{E} 1 / \mathrm{E} 3$ coexpression (Figure $1, \mathrm{~b}$ and $\mathrm{c}$ ).

To test whether the latter results depend critically on the stoichiometry of expression, we used three different cDNA molar ratios in the transient transfections. As shown in Figure 2, activation times were approximately two orders of magnitude smaller when E3 was present, independent of the cDNA molar ratios used. The activation time constant was significantly larger at the highest Q1/E1/E3 cDNA molar ratio (6:6:1) than at the lowest ratio of $1: 1: 1(P<0.05)$, while there was no statistical difference between the other groups when E3 was present. These findings demonstrate that Q1 is highly sensitive to E3, independent of E1's presence.

Ex vivo expression of KCNE3 in native cells. Preliminary studies were done on isolated rabbit myocytes that were transduced in primary culture by AdCGI-KCNE3 to determine the effects of $\mathrm{E} 3$ on APD. Time to $90 \%$ repolarization $\left(\mathrm{APD}_{90}\right)$ was significantly reduced to $225 \pm 39$ milliseconds (range 78-340) in the E3-transduced myocytes $(n=6)$, compared with $457 \pm 29$ milliseconds

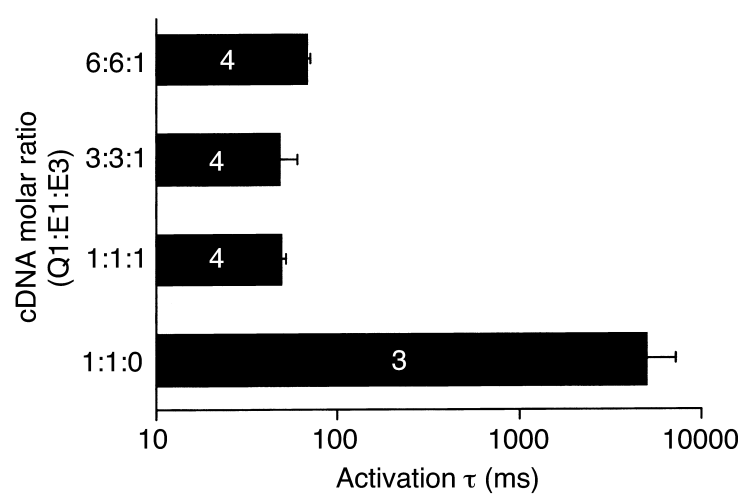

Figure 2

Effects of KCNE3 on activation kinetics of $I_{K}$ in transfected $\mathrm{CHO}$ cells. Activation rates $(\tau)$ at $40 \mathrm{mV}$ for different $\mathrm{cDNA}$ molar ratios. Note the logarithmic scale in activation rate for comparison. The number in each bar indicates the number of cells in each group. Q1, KCNQ1; E1, KCNE1; E3, KCNE3. 
a

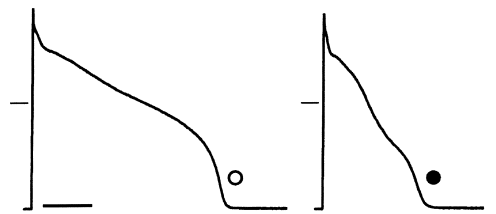

b

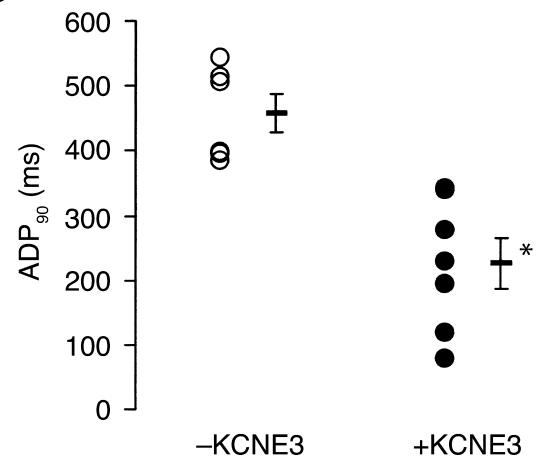

Figure 3

Effects of KCNE3 on APD; ex vivo transduction of rabbit ventricular myocytes in primary culture. (a) Representative traces of action potential and (b) pooled data of $\mathrm{APD}_{90}$ from control $(-\mathrm{KCNE3}, n=7$, open symbols) and E3-transduced (+KCNE3, $n=6$, filled symbols) myocytes, at $1 \mathrm{~Hz}$ pacing cycle. Scale bar $=0.1$ seconds; dash marks designate $0 \mathrm{mV}$. ${ }^{*} P<0.05$ vs. control.

$(P<0.05)$ in control cells $(n=7)$, while time to $50 \%$ repolarization $\left(\mathrm{APD}_{50}\right)$ was reduced from $222 \pm 43$ milliseconds to $128 \pm 30$ milliseconds $(P<0.05)$ at $1 \mathrm{~Hz}$ pacing rate (Figure 3 ). Similar results were obtained for 0.5 and $2 \mathrm{~Hz}$ pacing rates (data not shown). There was no change in the resting membrane potential between the two groups ( $-65.6 \pm 1.0$ vs. $-64.5 \pm 1.7 \mathrm{mV}$, E3-transduced vs. control).

In vivo expression of KCNE3. To investigate the effects of $\mathrm{E} 3$ expression on action potential repolarization, E3 was injected into the left ventricles of guinea pigs. Ectopic expression of E3 in cardiac myocytes resulted in an augmentation in action potential repolarization and therefore a reduction in APD (Figure 4). $\mathrm{APD}_{90}$ was reduced significantly from $287 \pm 19$ milliseconds in control myocytes $(n=7)$ to $98 \pm 8$ milliseconds in E3-transduced myocytes $(n=6)(P<0.05)$ at $1 \mathrm{~Hz}$ pacing rate. Likewise, $\mathrm{APD}_{50}$ was reduced from $250 \pm 19$ milliseconds in control cells to $65 \pm 8$ milliseconds in E3-transduced myocytes $(P<0.05)$. Similar trends were observed for both $\mathrm{APD}_{50}$ and $\mathrm{APD}_{90}$ at 0.5 and $2 \mathrm{~Hz}$ pacing rates (data not shown). Expression of E3 did not alter the resting membrane potential of cardiac myocytes ( $-71 \pm 1$ vs. $-68 \pm 2 \mathrm{mV}$, control vs. E3-transduced), or the cell capacitance ( $110 \pm 8$ vs. $101 \pm 5 \mathrm{pF}$, control [ $n=9]$ vs. E3-transduced [ $n=8]$ ).

Since coexpression of E3 with HERG reduced HERG current in oocytes (6), we anticipated that E3 might interact with HERG to reduce $\mathrm{I}_{\mathrm{Kr}}$ density in cardiomyocytes. We tested this idea indirectly by examining the effects of an $\mathrm{I}_{\mathrm{Kr}}$ blocker (E-4031) (23) on APD in control and E3-transduced myocytes. We found that the addition of $5 \mu \mathrm{mol} / 1 \mathrm{E}-4031$ to the bath solution had no significant effect on APD in E3-transduced myocytes $\left(\mathrm{APD}_{90}\right.$ of $98 \pm 8$ milliseconds when E-4031 was absent compared with $108 \pm 10$ milliseconds when it was present) (Figure 4b). Conversely, E-4031 increased $\mathrm{APD}_{50}$ and $\mathrm{APD}_{90}$ by approximately 87 and 88 milliseconds, respectively, in control cells (Figure $4 \mathrm{~b})$. The finding that E3-transduced cells are resistant to E-4031 suggests that E3 may functionally interact with HERG in cardiac myocytes to reduce $\mathrm{I}_{\mathrm{Kr}}$; nevertheless, the acceleration and enhancement of Q1 current predominates and produces net abbreviation of the action potential.

To test this notion and specifically to determine whether the abbreviation in APD is due to an increase in $\mathrm{I}_{\mathrm{Ks}}$, we recorded currents in E3-transduced myocytes in the presence of $5 \mu \mathrm{mol} / \mathrm{l} \mathrm{E}-4031$ to block $\mathrm{I}_{\mathrm{Kr}}$. As in $\mathrm{CHO}$ cells, expression of $\mathrm{E} 3$ in native cardiomyocytes increased both the density and the rate of activation of $\mathrm{I}_{\mathrm{Ks}}$ (Figure 5). $\mathrm{I}_{\mathrm{Ks}}$ density measured after 600 milliseconds of activation was on average sixfold larger in E3transduced myocytes than in control myocytes (Figure $5 \mathrm{~b}$ ), while it was about fourfold larger after 5000 milliseconds of activation (Figure $5 \mathrm{c}$ ). Rate of activation, determined by the ratio of currents at 5000 milliseconds to 600 milliseconds of activation, was significantly increased in E3-transduced myocytes compared with control cells (Figure 5d). This ratio was close to unity in the E3-transduced cells, indicating the presence of a time-independent current presumably as a result of interaction between $\mathrm{Q} 1$ and $\mathrm{E} 3$ in native cells.
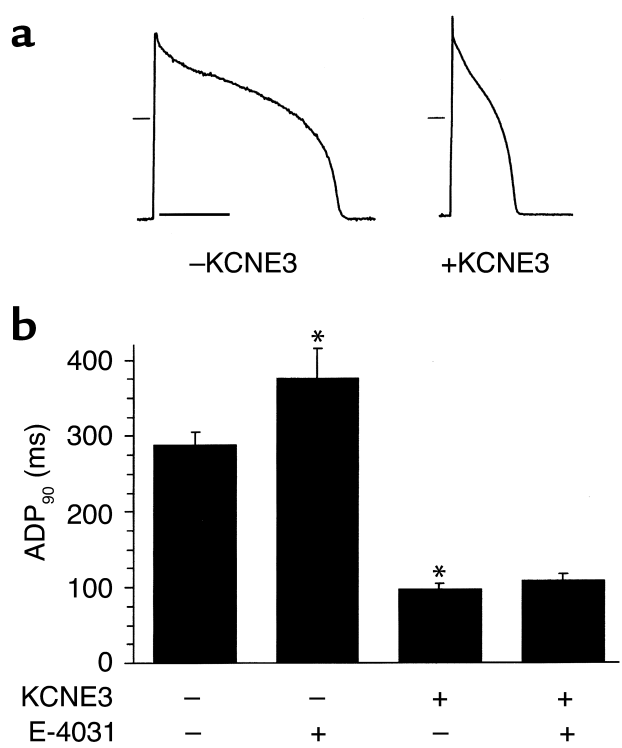

\section{Figure 4}

Effects of KCNE3 on APD; in vivo transduction of guinea pig ventricular myocytes. (a) Representative traces of action potential in control (-KCNE3) and E3-transduced (+KCNE3) myocytes. (b) Pooled data of $A P D_{90}$ from control and E3-transduced myocytes, in the presence and absence of $5 \mu \mathrm{mol} E-4031$ in the bath solution, at $1 \mathrm{~Hz}$ pacing cycle. Scale bar $=0.1$ seconds; dash marks designate 0 $\mathrm{mV}$. ${ }^{*} P<0.05$ vs. control $(-\mathrm{KCNE} 3,-\mathrm{E}-4031)$. 


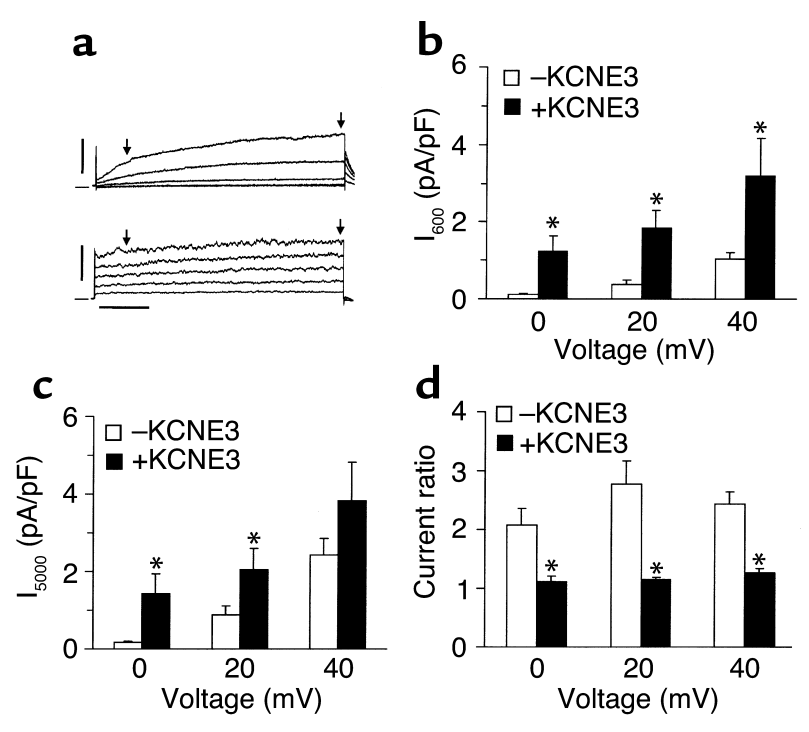

Consistent with action potential recordings in isolated E3-transduced myocytes, the QT interval of the ECG in E3-transduced animals was abbreviated significantly compared with baseline (QTc of $158 \pm 4$ milliseconds at baseline vs. $143 \pm 4$ milliseconds at day $3, n=6 ; P<0.05$ ). In these animals, RR interval was also shortened from $232 \pm 4$ milliseconds at baseline to $207 \pm 5$ milliseconds at day $3(P<0.05)$, with no change in PR interval. There was no observable change in the T wave or QRS-complex morphology. To ensure that these observations were not a consequence of adenoviral exposure itself and were entirely due to the presence of E3, we transduced two guinea pigs with AdCGI adenovirus, which is the same construct as AdCGI-KCNE3 but lacking the $\mathrm{E} 3$ gene. In neither animal did we observe changes in the QT or RR interval (e.g., QTc of $149 \pm 10$ and $154 \pm 7$ milliseconds, for baseline and 72 hours after injection, respectively), indicating that shortening of QT interval in animals transduced with adenovirus carrying E3 reflects an underlying reduction in APD in the E3-transduced cardiomyocytes.

While APDs measured in the E3-transduced myocytes were reduced on average by $66 \%$, we only observed a $10 \%$ reduction in QT interval (QTc) in

\section{Figure 6}

Mathematical model of action potential propagation in left ventricular tissue. (a) Upper panel: Geometry of the cable model used, based on a portion of the left ventricular wall. Middle panel: Representative action potential traces in endocardial (Endo), midwall (Mid), and epicardial (Epi) "myocytes" (or nodes). Action potential propagation was initiated at the endocardial surface, in a $3 \times 3$ area. Lower panel: The corresponding ECG. Horizontal scale bars $=0.1$ seconds. (b) Predicted relation between repolarization time (Repol.; activation time $\left.+\mathrm{APD}_{90}\right)$ and $\mathrm{APD}_{90}$ calculated at each layer from the corresponding action potential with respect to transmural depth. (c) Predicted relation between KCNE3 transduction rate and percent change in the simulated QT interval (\% $\Delta \mathrm{QT})$ relative to $0 \%$ transduction model. Note that the experimental finding (open square, mean \pm SEM) lies on this curve. APD 90 , time to $90 \%$ repolarization.

\section{Figure 5}

Effects of KCNE3 on $I_{K}$; in vivo transduction of guinea pig ventricular myocytes. (a) Original current traces of E-4031-insensitive delayed rectifier currents (i.e., $\mathrm{I}_{\mathrm{Ks}}$ ) recorded with 5 -second depolarizing steps from -20 to $60 \mathrm{mV}$ in $20-\mathrm{mV}$ increments, followed by a repolarizing pulse to $-50 \mathrm{mV}$ for 0.3 seconds, with 10 -second repetition cycles in a control (upper panel) and an E3-transduced (lower panel) myocyte. Horizontal scale bar $=1$ second; vertical scale bar $=2.5$ and $5.0 \mathrm{pA} / \mathrm{pF}$ for upper and lower panels, respectively. ( $b$ and $\mathbf{c}$ ) Current densities after 600 milliseconds (b) and 5000 milliseconds (c) activation (arrows in a) at three different voltages in control (-KCNE3, $n=8$ ) and E3-transduced (+KCNE3, $n=8$ ) cells. (d) Ratio of current densities at 5000 milliseconds relative to 600 milliseconds of activation. ${ }^{*} P<0.05$ vs. control $(-\mathrm{KCNE} 3)$.

transduced animals. This discrepancy between reductions in APD in isolated cells and QT interval of the ECG is primarily due to the transduction efficiency. In these studies, we estimated a $10-20 \%$ transduction rate - as determined by percentage of GFP-transduced cells after isolation. Therefore, one would expect that action potential abbreviations in isolated cells would not translate to similar percentages of QT interval abbreviation on the ECG, simply due to the

$\mathbf{a}$

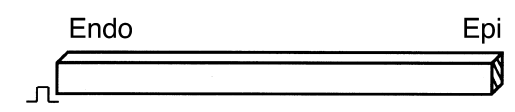

b
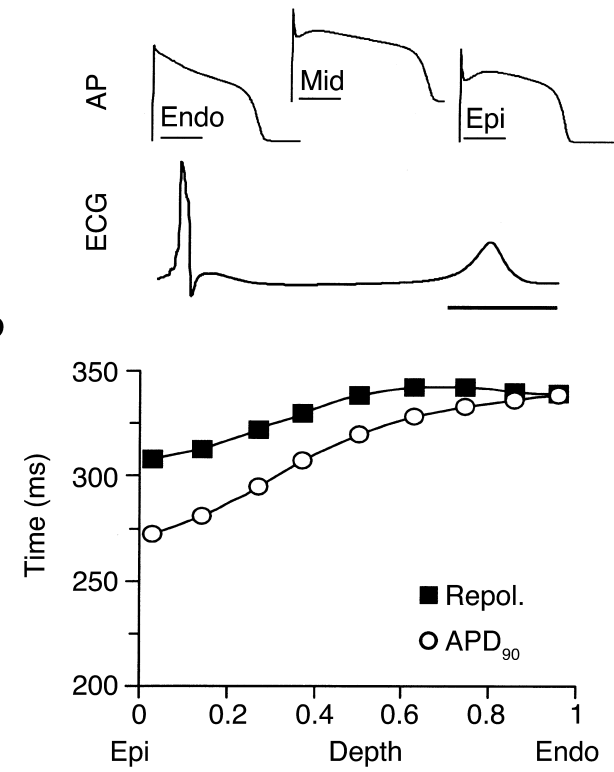

C

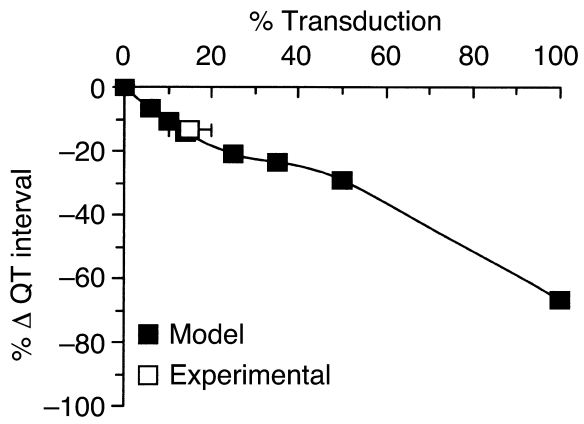




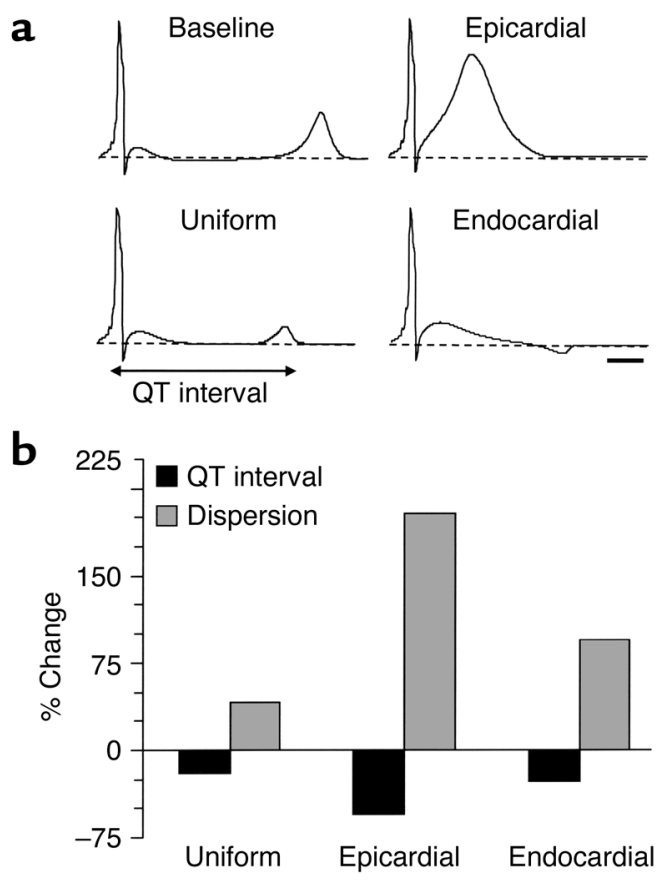

\section{Figure 7}

Effects of heterogeneous KCNE3 transduction on the simulated ECG. (a) Simulated ECGs for baseline, 25\% uniform transmural transduction (Uniform), 25\% of "myocytes" only in the epicardial region (Epicardial), and $25 \%$ of "myocytes" only in the endocardial region (Endocardial). Horizontal scale bar $=50$ milliseconds. (b) Predicted percent change in QT interval of the simulated ECG and dispersion of repolarization, calculated from the difference in the highest and lowest repolarization time (activation time $+\mathrm{APD}_{90}$ ) across the ventricular wall, relative to baseline model.

electrical coupling between E3-transduced and nontransduced myocytes. To check for quantitative consistency in this argument, we used a mathematical model of cardiac action potential propagation in the heart (18), where a variable percentage of the nodes or "myocytes" possessed an additional ionic current modeled to mimic that associated with Q1-E3 coassembly (Figure 6a). The baseline model reproduces transmural repolarization time $\left(\mathrm{APD}_{90}+\right.$ conduction time) and action potential durations ( $\left.\mathrm{APD}_{90}\right)$ measured experimentally (24). We found that, similar to our experimental observations, a $15 \%$ uniform transmural transduction rate resulted in $14 \%$ reduction in the simulated QT interval in the model (Figure $6 c$ ), which is within one SEM of our experimental observations. More importantly, the model showed that, in order to achieve a reduction in QT interval comparable to the APD abbreviation seen in isolated cells (66\%), a transduction rate of approximately $70 \%$ or higher is needed (Figure 6c).

While ectopic E3 expression can abbreviate the QT interval both in vivo and in silico, such expression might actually destabilize repolarization if marked heterogeneities occur. Locally intense transduction of one region or transmural layer could, in principle, lead to such heterogeneities and predispose to arrhythmias. In transmural wedge preparations, the "dispersion of repolarization," defined as the difference between the longest and shortest repolarization time (activation time $+\mathrm{APD}_{90}$ ) across the ventricular wall, has been validated as a sensitive marker of repolarization heterogeneity $(24,25)$. To test the effects of heterogeneous expression of $\mathrm{E} 3$ in relation to arrhythmias, we numerically expressed E3 in the $25 \%$ outer (epicardial) portion of the model; such a distribution mimics that achieved with some adenoviral delivery methods (26). While QT interval of the model ECG was reduced to a greater degree than with $25 \%$ uniform transmural transduction (Figure 7, $a$ and $b$ ), the dispersion of repolarization across the ventricular wall was actually elevated (Figure 7 , a and b). Similar findings were obtained when E3 was expressed only in the $25 \%$ inner (endocardial) portion of the model (Figure 7, a and b). However, endocardial expression resulted in an elevation in the ST segment and an inverted $\mathrm{T}$ wave on the simulated ECG (Figure 7a). This indicates the importance of homogeneous transmural E3 transduction across the ventricular wall.

\section{Discussion}

In this study we expressed an accessory subunit, E3, which is not normally expressed in left ventricular tissue, by means of somatic gene transfer to regulate action potential repolarization. In $\mathrm{CHO}$ cells, we demonstrated that $\mathrm{E} 3$ coassembles with Q1 in the presence or absence of $\mathrm{E} 1$ and results in a channel that activates instantaneously and is open at all measured voltages. Ectopic expression of E3 in cardiac myocytes both ex vivo and in vivo resulted in a significant reduction in APD. Shortening of the action potential in myocytes transduced in vivo was consistent with a significant increase in $\mathrm{I}_{\mathrm{Ks}}$, which activated more rapidly and at more hyperpolarized voltages compared with control cells. More importantly, overexpression of E3 resulted in a reduction in the QT interval of the ECG in the transduced animals, parallel to the results from isolated E3-transduced myocytes.

Results in CHO cells suggest that Q1 is more sensitive to $\mathrm{E} 3$ than it is to E1, independent of the molar ratio of cDNA expressed in these cells. Wang et al. (27), by taking advantage of fusion protein channels, put forward the possibility of multiple Q1-E1 stoichiometries. Based on these observations, it is likely that even the presence of one KCNE peptide - in this case E3 - out of a possible four (28) coassembled with a Q1 homotetramer could alter the activation properties and regulate the current expressed, as seen here and by others $(6,29)$.

Preliminary studies done on rabbit myocytes, transduced with AdCGI-KCNE3 vector in culture, resulted in action potentials that were significantly shorter than in control cells. APDs observed in control cells after 24 hours of culture were, on average, longer than normal reported rabbit APDs (30). This is due to the downregulation of inward rectifier and transient outward potassium currents in cultured 
myocytes (30, 31). Mitcheson et al. (30) reported a 50millisecond increase in $\mathrm{APD}_{80}$, and depolarization of the resting membrane potential in rabbit myocytes after 24 hours of culture, attributed to the alterations in ionic currents measured. Similarly, we observed a slight depolarization $(\sim 5 \mathrm{mV})$ in the resting membrane potential in cultured rabbit myocytes compared with freshly isolated cells, independent of adenoviral transduction.

AdCGI-KCNE3 virus was also delivered in vivo and resulted in a significant reduction in the APD in freshly isolated E3-transduced myocytes. This was consistent with an observed approximately fivefold increase in $\mathrm{I}_{\mathrm{Ks}}$ in the transduced cells. The augmentation in $\mathrm{I}_{\mathrm{Ks}}$ was consistent at all voltages measured and particularly at voltages in the plateau range of the action potential, indicating that this current (because of its rapid activation) would be active throughout phases 2 and 3 of the action potential, unlike native $\mathrm{I}_{\mathrm{Ks}}$, which is active predominantly in phase 3.

In vivo delivery of AdCGI-KCNE3 in guinea pigs also resulted in a significant reduction in the QT interval of the ECG of transduced animals. While APDs measured in the E3-transduced myocytes were reduced on average by $66 \%$, we only observed a $10 \%$ reduction in QT interval (QTc) in transduced animals. Quantitative modeling rationalized the degree of QT interval abbreviation as a consequence of electrotonic interactions in the face of limited transduction efficiency. More importantly, the model showed that, in order to achieve a reduction in QT interval comparable to the APD abbreviation seen in isolated cells (66\%), a transduction rate of approximately $70 \%$ or higher is needed (Figure 6c). Nevertheless, the present simulations point out one potential benefit of low transduction efficiency in the heart - excessive electrical effects are dampened by electrotonic interactions with nontransduced cells in the cardiac syncytium, so as to produce therapeutically relevant alteration of global parameters.

A potential pitfall of limited transduction efficiency is the heterogeneity of repolarization that may occur with locally intense but patchy expression. Adenoviral delivery methods (e.g., ref. 26) can result in much more pronounced transduction efficiency in the subepicardium than in other layers. This could cause a problem in the case of E3 transduction, since a high level of $\mathrm{I}_{\mathrm{Ks}}$ heterogeneity across the ventricular wall could result in abnormal repolarization patterns - in this case, more pronounced in epicardium than in other layers - serving as a precursor to arrhythmias. To address this issue, we numerically restricted the electrophysiological consequences of $\mathrm{E} 3$ transduction to the $25 \%$ most epicardial nodes of the model. This resulted in a greater abbreviation of the QT interval of the simulated ECG compared with homogeneous transmural transduction, while increasing the degree of transmural dispersion of repolarization by $200 \%$ (Figure 7). This paradox (shorter QT, but greater disper- sion of repolarization) re-emphasizes the significance of the adenoviral delivery method used; recent approaches to viral particle delivery, as used here and previously by others $(32,33)$, result in a more transmurally uniform transduction across the ventricular wall. Nevertheless, the details of transgene distribution will critically determine the eventual efficacy of gene therapy for LQT syndrome.

In summary, we have shown that the introduction of accessory subunits, such as E3, that coassemble with channels in the heart through somatic gene transfer and regulate their function could be exploited as a therapeutic option for patients with LQT syndrome. E3-transduced cells had a shorter APD than control cells, while E-4031-insensitive $\mathrm{I}_{\mathrm{K}}$ and activation kinetics of E3-transduced cells were significantly augmented compared with control cells. Thus, expression of E3 increases outward current and abbreviates APD in cardiac myocytes. Since E3 is not normally expressed in ventricular tissue, it is potentially an attractive candidate for gene therapy for disorders associated with abnormal cardiac repolarization, at least at a conceptual level. It has been proposed that HERG overexpression through somatic gene transfer may be useful for stabilization of cardiac repolarization in LQT syndrome patients (12). However, HERG mutations are common in LQT syndrome (LQT2) (3), and many possess dominant negative features. Therefore, overexpression of wild-type HERG in such patients may not be very effective. Since E3 significantly reduces $\mathrm{I}_{\mathrm{Kr}}$, possibly through interaction with HERG, while augmenting total outward $\mathrm{I}_{\mathrm{K}}$ and enhancing action potential repolarization, E3 may be a more attractive candidate for correction of repolarization in patients with HERG mutations. However, in patients with Q1 mutation(s) (LQT1), where most of the genetic lesions are dominant negative as well, this approach may not be as effective. In any case, our modeling results highlight the importance of relatively even transgene distribution in order to achieve salutary QT interval abbreviation without undermining repolarization. Further studies in animal models of LQT syndrome and heart failure will be required to assess the ability of E3 to enhance cardiac repolarization without adversely affecting other properties.

\section{Acknowledgments}

This work was supported by NIH/National Heart, Lung, and Blood Institute Specialized Center of Research grant P50 HL-52307 (to E. Marbán), the American Heart Association (AHA) Scientist Development Grant (to H.B. Nuss), and NIH grant RO1 HL-66381 (to H.B. Nuss). Modeling efforts were supported by NIH grant RO1 HL-60133 (to R.L. Winslow). R. Mazhari was supported by NIH postdoctoral training grant HL-O7227. A.A. Armoundas was supported by an AHA postdoctoral fellowship (0020257U) from the Mid-Atlantic Affiliate. 
The authors thank M. Estevez, J. Seharaseyon, M. Leppo, A. McDonald, A. Janssen, and C. Yung for their valuable technical assistance, and J. Miake for helpful discussions. We thank Eisai Co. (Tokyo, Japan) for kindly providing E-4031. E. Marbán holds the Michel Mirowski, M.D. Professorship of Cardiology of the Johns Hopkins University.

1. Keating, M.T., and Sanguinetti, M.C. 2001. Molecular and cellular mechanisms of cardiac arrhythmias. Cell. 104:569-580.

2. Roden, D.M. 1998. Mechanisms and management of proarrhythmia. Am. J. Cardiol. 82:49I-57I

3. Splawski, I., et al. 2000. Spectrum of mutations in long-QT syndrome genes. KVLQT1, HERG, SCN5A, KCNE1, and KCNE2. Circulation. 102:1178-1185.

4. Splawski, I., Tristani-Firouzi, M., Lehmann, M.H., Sanguinetti, M.C., and Keating, M.T. 1997. Mutations in the hminK gene cause long QT syndrome and suppress IKs function. Nat. Genet. 17:338-340.

5. Abbott, G.W., et al. 1999. MiRP1 forms IKr potassium channels with HERG and is associated with cardiac arrhythmia. Cell. 97:175-187.

6. Schroeder, B.C., et al. 2000. A constitutively open potassium channel formed by KCNQ1 and KCNE3. Nature. 403:196-199.

7. Abbott, G.W., et al. 2001. MiRP2 forms potassium channels in skeletal muscle with Kv3.4 and is associated with periodic paralysis. Cell. 104:217-231.

8. Bleich, M., and Warth, R. 2000. The very small-conductance $\mathrm{K}+$ channel KvLQT1 and epithelial function. Pflugers Arch. 440:202-206.

9. Franco, D., et al. 2001. Divergent expression of delayed rectifier $\mathrm{K}(+)$ channel subunits during mouse heart development. Cardiovasc. Res. 52:65-75.

10. Boucherot, A., Schreiber, R., and Kunzelmann, K. 2001. Regulation and properties of KCNQ1 $(\mathrm{K}(\mathrm{V}) \mathrm{LQT} 1)$ and impact of the cystic fibrosis transmembrane conductance regulator. J. Membr. Biol. 182:39-47.

11. Hoppe, U.C., Marbán, E., and Johns, D.C. 2000. Adenovirus-mediated inducible gene expression in vivo by a hybrid ecdysone receptor. Mol. Ther. 1:159-164

12. Hoppe, U.C., Marbán, E., and Johns, D.C. 2001. Distinct gene-specific mechanisms of arrhythmia revealed by cardiac gene transfer of two long QT disease genes, HERG and KCNE1. Proc. Natl. Acad. Sci. USA. 98:5335-5340

13. Hardy, S., Kitamura, M., Harris-Stansil, T., Dai, Y., and Phipps, M.L. 1997. Construction of adenovirus vectors through Cre-lox recombination. J. Virol. 71:1842-1849.

14.Johns, D.C., Marx, R., Mains, R.E., O’Rourke, B., and Marbán, E. 1999. Inducible genetic suppression of neuronal excitability. J. Neurosci. 19:1691-1697.

15. Hajjar, R.J., del Monte, F., Matsui, T., and Rosenzweig, A. 2000. Prospects for gene therapy for heart failure. Circ. Res. 86:616-621.

16. Seharaseyon, J., et al. 2000. Molecular composition of mitochondrial
ATP-sensitive potassium channels probed by viral Kir gene transfer. J. Mol. Cell. Cardiol. 32:1923-1930.

17. Hayes, E., Pugsley, M.K., Penz, W.P., Adaikan, G., and Walker, M.J. 1994 Relationship between QaT and RR intervals in rats, guinea pigs, rabbits, and primates. J. Pharmacol. Toxicol. Methods. 32:201-207.

18. Winslow, R.L., et al. 2000. Electrophysiological modeling of cardiac ventricular function: from cell to organ. Annu. Rev. Biomed. Eng. 2:119-155.

19. Winslow, R.L., Rice, J., Jafri, S., Marbán, E., and O’Rourke, B. 1999. Mechanisms of altered excitation-contraction coupling in canine tachycardiainduced heart failure. II. Model studies. Circ. Res. 84:571-586.

20. Liu, D.W., Gintant, G.A., and Antzelevitch, C. 1993. Ionic bases for electrophysiological distinctions among epicardial, midmyocardial, and endocardial myocytes from the free wall of the canine left ventricle. Circ. Res. 72:671-687.

21. Zygmunt, A.C., Goodrow, R.J., and Antzelevitch, C. 2000. I(NaCa) contributes to electrical heterogeneity within the canine ventricle. Am.J. Physiol. Heart Circ. Physiol. 278:H1671-H1678.

22. Sanguinetti, M.C., et al. 1996. Coassembly of K(V)LQT1 and minK (IsK) proteins to form cardiac I(Ks) potassium channel. Nature. 384:80-83.

23. Sanguinetti, M.C., and Jurkiewicz, N.K. 1990. Two components of cardiac delayed rectifier $\mathrm{K}+$ current. Differential sensitivity to block by class III antiarrhythmic agents. J. Gen. Physiol. 96:195-215.

24. Yan, G.X., Shimizu, W., and Antzelevitch, C. 1998. Characteristics and distribution of $\mathrm{M}$ cells in arterially perfused canine left ventricular wedge preparations. Circulation. 98:1921-1927.

25. Yan, G.X., and Antzelevitch, C. 1998. Cellular basis for the normal T wave and the electrocardiographic manifestations of the long-QT syndrome. Circulation. 98:1928-1936.

26. Maurice, J.P., et al. 1999. Enhancement of cardiac function after adenoviral-mediated in vivo intracoronary beta2-adrenergic receptor gene delivery. J. Clin. Invest. 104:21-29.

27. Wang, W., Xia, J., and Kass, R.S. 1998. MinK-KvLQT1 fusion proteins, evidence for multiple stoichiometries of the assembled IsK channel. J. Biol. Chem. 273:34069-34074.

28. Abbott, G.W., and Goldstein, S.A. 1998. A superfamily of small potassium channel subunits: form and function of the MinK-related peptides (MiRPs). Q. Rev. Biophys. 31:357-398.

29. Melman, Y.F., Domenech, A., de la Luna, S., and McDonald, T.V. 2001 Structural determinants of KvLQT1 control by the KCNE family of proteins. J. Biol. Chem 276:6439-6444.

30. Mitcheson, J.S., Hancox, J.C., and Levi, A.J. 1996. Action potentials, ion channel currents and transverse tubule density in adult rabbit ventricular myocytes maintained for 6 days in cell culture. Pflugers Arch. 431:814-827.

31. Mitcheson, J.S., Hancox, J.C., and Levi, A.J. 1998. Cultured adult cardiac myocytes: future applications, culture methods, morphological and electrophysiological properties. Cardiovasc. Res. 39:280-300.

32. Hajjar, R.J., et al. 1998. Modulation of ventricular function through gene transfer in vivo. Proc. Natl. Acad. Sci. USA. 95:5251-5256.

33. Shah, A.S., et al. 2000. Intracoronary adenovirus-mediated delivery and overexpression of the beta(2)-adrenergic receptor in the heart: prospects for molecular ventricular assistance. Circulation. 101:408-414. 